\title{
Bio-inspired Frequency Agile Acoustic System
}

\author{
José Guerreiro, Joseph C. Jackson and James F.C. Windmill \\ Centre for Ultrasonic Engineering \\ University of Strathclyde \\ Glasgow, Scotland \\ jose.guerreiro@strath.ac.uk
}

\begin{abstract}
Natural passive mechanical systems such as ear tympanic membranes may show active responses by incorporating feedback mechanisms which then affect their mechanical structure. In this paper, the moth's auditory system is used as a biological model of inspiration. A smart acoustic system which alters its natural resonance frequency was developed. Experimental results, given by a proposed-built real-time embedded system, show time and amplitude dependency towards dynamic frequency adaptation according to the intensity of acoustic input signals.
\end{abstract}

Keywords-bio-inspired acoustic transducer; dynamic frequency adaptation; feedback control; embedded system.

\section{INTRODUCTION}

Bio-systems are a result of millions of years of evolutionary processes that make them very power efficient and well adapted to perform their role in a living body. The synergy between engineering and biology landscapes can be of great value. On the one hand, engineering is looking into biology to get inspiration to design optimised and better systems to measure and process signals [1]. On the other hand, engineering is used as a tool, for instance to improve damaged biological systems by using prostheses [2][3]. Biological periphery sensors possess clever mechanisms to pre-process and compress information before sending it to high level computational systems (e.g. the brain). Auditory systems are a good example of that, exhibiting many ways to compute acoustic information such as frequency selectivity (tuning), temporal coding (directional cues) and amplification (adaptation for different environments). Insect hearing has been used widely as a model system of research due to its reduced complexity compared with mammals, and examples of novel designs of microphones have been presented as outcomes of bio-inspiration [4][5][6]. This work presents a preliminary prototype of an electromechanical acoustic transducer with a feedback control system which allows dynamic adaptation of its natural frequency response, bioinspired by the auditory system of moths (Fig. 1a).

\section{BIO-SYSTEM MODEL AS INSPIRATION}

\section{A. Moth's Auditory System}

Moths have a tympanum-like ear and they can hear within a broad spectrum of frequencies, from $10 \mathrm{kHz}$ up to $300 \mathrm{kHz}$ [7]. However, for quiet sounds, the Noctuid moth shows higher sensitivity at low frequencies (Fig 1c - green trace). Additionally, within an imminent attack of its biggest predator (bats), that sensitivity at low frequencies is shifted towards higher frequencies (Fig 1c - orange trace). Literature suggests

This research is funded by the European Research Council under the European Union's Seventh Framework Programme (FP/2007-2013) / ERC Grant Agreement n. [615030]. this dynamic tuning response (Fig $1 \mathrm{~b}$ ) is a defence mechanism in order to become more sensitive to high frequency echolocation calls emitted by bats signalling the final stage of hunting - once relatively close to its target [8]. This shift up to higher frequencies may enhance neuronal activity to enable the moths to trigger behavioural responses to escape from a fatal predation. Active feedback processes (neuronal processing) may play a fundamental role in the mechanism for dynamically changing the natural resonance frequency in the auditory system.

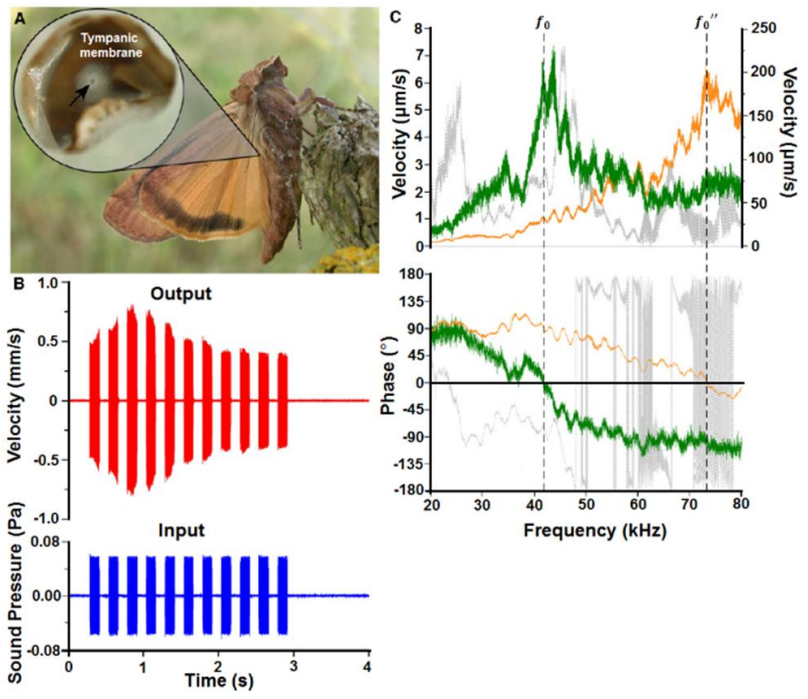

Fig. 1 - Tympanic membrane tuning response in the moth Noctua pronuba. (A) Microscale ear of N. pronuba. (B) Mechanical response (red trace) of the tympanic membrane to bat-like incoming sounds (blue trace). (C) Frequency response of the tympanic membrane for low intensity stimulus (green trace) and for high-intensity stimulus (orange trace). Redrawn and adapted with kind permission from [8].

\section{B. Modelling of a Biological Acoustic Transducer from the Engineering Point of View}

From the engineering point of view, it might be hypothesised that the tympanic membrane is seen as a mass-spring system (e.g. simple harmonic oscillator - SHO) expressed by (1), with natural resonance frequency given by the ratio between stiffness and mass $\left(\omega_{0}=\sqrt{\mathrm{k} / \mathrm{m}}\right)$, quality factor is expressed by the ratio between resonance frequency and damping term $\left(Q=\omega_{0} / \gamma\right)$, where $k$ represents the spring factor (i.e. stiffness), $m$ is mass, $b$ is the component losses coupled to the system and $F \cos (\omega t)$ represents an external force added to the system (e.g. a sound pressure signal). 


$$
m \ddot{x}+b \dot{x}+k x=F \cos (\omega t)
$$

The natural resonance frequency is directly proportional to the spring's stiffness and mass which by modelling one of these elements (reactive terms) will allow the frequency response of the system to be altered. Furthermore, Q is also dependent on the natural resonance frequency of the system. It is reported in [8] that the tympanic membrane of Noctuid moths might be somehow mechanically stretched to allow a dynamic tuning adaptation. Stretching may be provided to this system by increasing tension over it (i.e. adding an external static force) which then expands the membrane and consequently will change its natural frequency response. From the engineering point of view, this exercise might be simply generalised as a beam (membrane) fixed on both sides while stretching is provided by adding a transverse tension at those points (e.g. pulling apart) which ideally expands the membrane uniformly and consequently alters the frequency response of the system, as illustrated in Fig. 2.

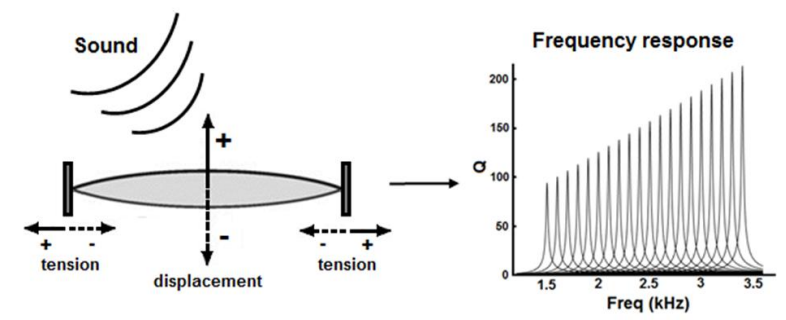

Fig. 2 - A fixed-fixed beam structure as a simple system to model the behaviour of the tympanic membrane of moths. Frequency response is changed by increasing tension.

In order to investigate and better understand the response given by this system, an electromechanical setup is presented. The transducer part of the system is made using a thin Kapton membrane (polyimide film - width $=22 \mathrm{~mm}$; height $=3 \mathrm{~mm}$; thickness $=50 \mu \mathrm{m})$ placed and glued on top of a piezoelectric (PZT) stack device which is used to provide a static force to expand and consequently stretch the Kapton membrane (Fig. 3a).
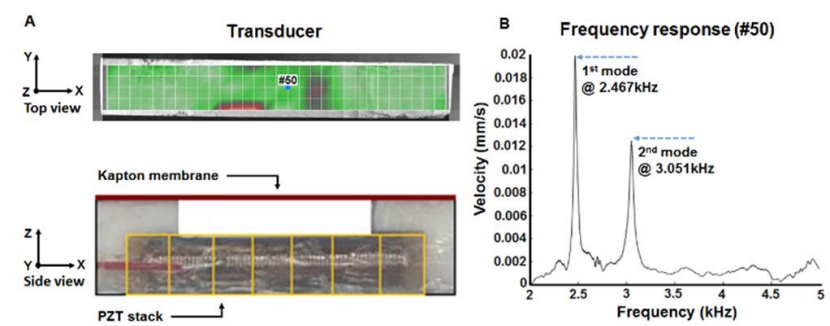

Fig. 3 - (A) Acoustic transducer built with a Kapton membrane and a PZT stack. (B) Frequency response of the transducer showing 2 resonance modes.

\section{Transducer Characterization}

In order to evaluate acoustic-structure interactions in the transducer part of the system, laser Doppler vibrometer (LDV) techniques were used. A full scan of the Kapton membrane was measured during acoustic stimuli. Outlining the frequency response in a central point (\#50) of the membrane, it shows multiple resonance modes (see Fig. 3b). As previously described, the resonance frequency may be adapted by adding an external static force which will increase tension over the Kapton membrane. This external force is provided by the PZT stack driven with a static DC voltage. The PZT stack used in this work was a PICMA $®$ stack multilayer piezo actuator $(\mathrm{P}-885.51)$ from PI Ceramic, with operating voltages up to $120 \mathrm{~V}$. Evaluation tests were conducted in order to analyse the frequency response while increasing the driving voltage of the PZT stack to increase the tension in the membrane. Driving voltages were provided with $5 \mathrm{~V}$ step increments, resulting in a proportional increase of the natural resonance frequency of the membrane, as shown in Fig. 4. It closely follows the theoretical frequency response of a SHO shown previously in Fig. 2.

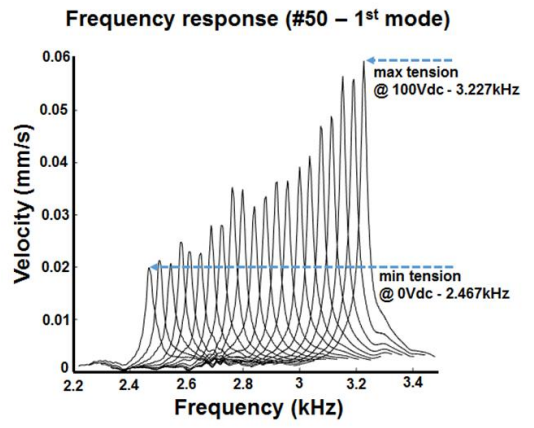

Fig. 4 - Frequency response of the Kapton membrane while providing different tensions. Driving voltage vs natural frequency response.

\section{FEEDBACK CONTROL SYSTEM}

Motivated by the dynamics and active mechanisms seen in the auditory system of moths, a feedback control system was developed to provide adaptation of the frequency response of the transducer. Fig. 5 presents a diagram overview of the feedback control system implemented, where the "Frequency Agile Sensor" block represents the transducer itself, and "Adapting Control System" is an Embedded System computing a threshold based algorithm used to dynamically adapt the mechanical properties of the transducer.

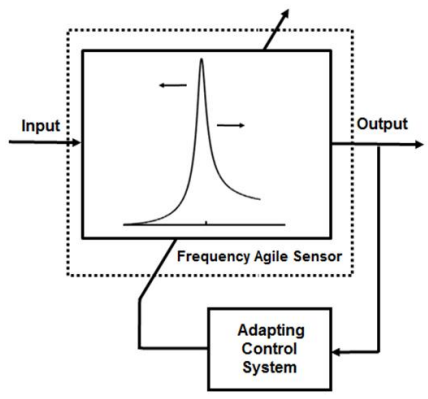

Fig. 5 - Diagram overview of the feedback control system used to achieve dynamic frequency adaptation.

\section{A. Embedded System - signal conditioning and acquisition platform}

In order to implement the system presented previously, it was essential to design an experimental setup (Fig. 6). As previously described, a Kapton membrane placed over a PZT stack is used as an acoustic transducer. A laser vibrometer (head + controller: Polytec OFV 2700) providing an analogue output was used as a reference measuring signal of the displacements 
induced onto the membrane from sound pressure signals. A Micro-controller Unit (MCU), STM32F4 running at $168 \mathrm{MHz}$, is used to compute a threshold based algorithm. Conditioning circuitry (low-noise amplifier and band-pass filtering implemented using standard off-the-shelf electronic components) was implemented for the laser output signals before acquisition using an on-board analogue-digital converter (12bit). Signals are acquired following interrupt based routines using a sampling frequency of $50 \mathrm{kHz}$. A threshold based algorithm running in the MCU is set according to the intensity of the input signals, such that the feedback system may adapt the mechanics of the membrane if the sound pressure signals presented are high enough to trigger the processing routines. In order to drive the PZT stack, a digital-analogue converter (12bit) is used and its output is amplified using a PZT driver circuit (power amplifier - using Apex Microtechnology PA78 device). The embedded system is designed to provide real-time results.

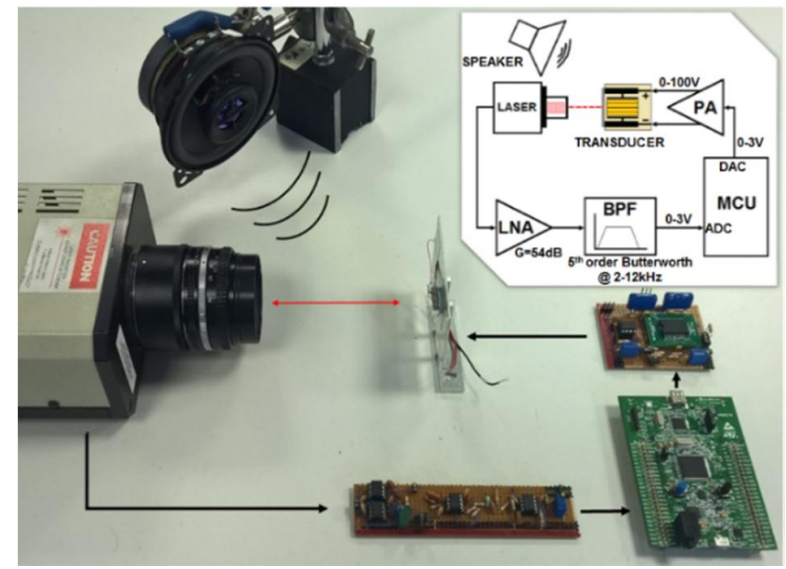

Fig. 6 - Setup of the embedded system used to implement the feedback control system in a real-time processing scenario.
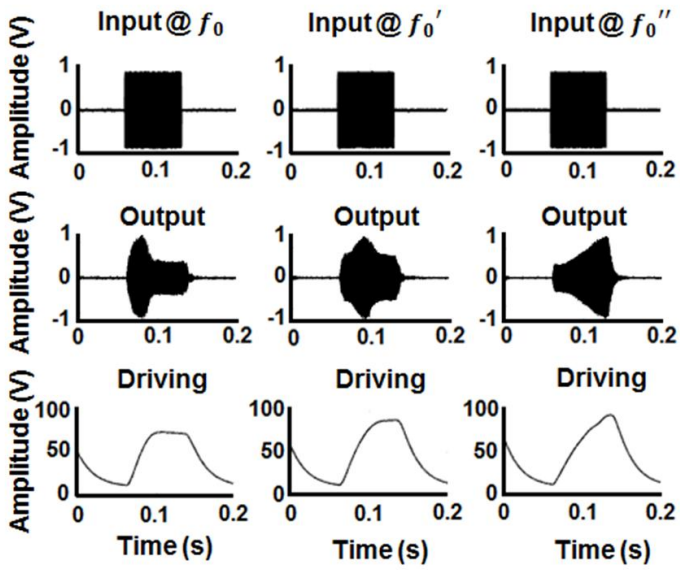

Fig. 7 - Dynamic frequency adaptation given by the bio-inspired system proposed: is Time $(0.1 \mathrm{~s})$ and Amplitude $(0.5 \mathrm{~V})$ dependent.

\section{B. Experimental Results}

Fig. 7 shows three different examples where the system adapts its natural frequency response showing time and amplitude dependency. Frequency tuning travels from $f_{0}$ towards $f_{0}^{\prime \prime}$. Firstly, it can be seen that the system is tuned at the beginning of the input signal with frequency $f_{0}$, however once tension is provided to the membrane it changes the mechanical properties consequently altering the frequency response - so that, it becomes off-resonant tuning after $0.1 \mathrm{~s}$. Secondly, the input frequency is above resonance with frequency $f_{0}{ }^{\prime}$, i.e. at this moment the natural resonance frequency of the system $\left(f_{0}\right)$ is not matched with the input frequency $\left(f_{0}{ }^{\prime}\right)$, however once tension is provided to it, frequency matching will occur once the input frequency and membrane's one are both the same at $f_{0}{ }^{\prime}$ (see similar response in Fig 1b reporting the tuning response in the Noctuid moth's tympanic membrane). Thirdly, the tuning response only appears when the membrane is fully expanded (max. tension) at $f_{0}{ }^{\prime \prime}$. With no acoustic input the feedback control system sets (min. tension) the membrane to return back to its initial natural frequency response.

\section{CONCLUSIONS}

This paper presents a study with experimental results of an implementation of a bio-inspired acoustic system. A smart acoustic transducer was created combining Kapton and piezoelectric ceramic materials with a feedback control system to enable dynamic adaptations of its frequency response. The natural resonance frequency is proportionally dependent with the amount of tension additionally supplied onto the passive system. The resulting tuning responses given by the presented system are time and amplitude dependent as seen within the natural system itself [8]. This new smart transducer concept provides real-time automatic adjustment of an acoustic sensor frequency response, which could have applications across different acoustic and ultrasonic fields requiring frequency agility and adaptive tuning.

\section{ACKNOWLEDGMENT}

The authors would like to thank Walter Galbraith and Jerzy Dziewierz for the useful discussions while the construction of the analogue circuits was integrated in the experimental setup and Roger Domingo-Roca for the 3D printing parts.

\section{REFERENCES}

[1] Sarpeshkar, R., "Ultra Low Power Bioelectonics: Fundamentals, Biomedical Applications, and Bio-inspired Systems". Cambridge University Press, 2010.

[2] Wee, K.H, Turicchia, L., and Sarpeshkar, R., "An Articulatory Silicon Vocal Tract for Speech and Hearing Prostheses". Biomedical Circuits and Systems, 5(4): 339-346, 2011.

[3] Sarpeshkar, R., Salthouse, C., Sit Ji-Jon, Baker, M.W., Zhak, S.M., Lu, T.K.-T, Turicchia, L., and Balster, S. “An ultra-low-power programmable analog bionic ear processor". Biomedical Engineering, 52(4):711-727, 2005.

[4] Miles, R.N., Hoy, R.R., "The Development of a Biologically-Inspired Directional Microphone for Hearing Aids". Audiology Neurotology, 11(2): 86-94, 2006.

[5] Reid, A., Windmill, J.F.C., and Uttamchandani, D., "Bio-Inspired Sound Localization Sensor with High Directional Sensitivity". Elsivier Procedia Engineering 120: 289-293, 2015.

[6] Zhang, Y., Bauer, R., Windmill, J.F.C., and Uttamchandani, D., "MultiBand Asymmetric Piezoelectric MEMS Microphone Inspired by the Ormia Ochracea". IEEE $29^{\text {th }}$ International Conference on MEMS, 11141117, 2016

[7] Moir, H.M., Jackson, J.C., and Windmill, J.F.C., "Extremely High Frequency Sensitivity in a 'Simple' Ear". Biology Letters, 2013.

[8] Windmill, J.F.C., Jackson, J.C., Tuck, E.J., and Robert, D., "Keeping up with Bats: Dynamic Auditory Tuning in a Moth". Current Biology 16: 2418-2423, 2006. 